\title{
Eradication of multidrug-resistant Acinetobacter baumannii in a female patient with total hip arthroplasty, with debridement and retention: a case report
}

\author{
Alison M Beieler*1, Robert W Belknap ${ }^{2}$, Michael R Dayton ${ }^{3}$, Connie S Price ${ }^{1}$ \\ and Steve J Morgan ${ }^{4}$
}

\begin{abstract}
Address: ${ }^{1}$ Department of Medicine, Infectious Diseases, Denver Health Medical Center, 660 Bannock St, Denver, CO 80204, USA, ${ }^{2}$ Department of Medicine \& Public Health, Infectious Diseases, Denver Health Medical Center, 605 Bannock St, Denver, CO 80204, USA, ${ }^{3}$ Department of Orthopedics, University of Colorado, 1635 N. Ursula St, Aurora, CO 80045, USA and ${ }^{4}$ Department of Orthopedics, Denver Health Medical Center, 777 Bannock St, Denver, CO 80204, USA
\end{abstract}

Email: Alison M Beieler* - alison.beieler@dhha.org; Robert W Belknap - robert.belknap@dhha.org;

Michael R Dayton - michael.dayton@uchsc.edu; Connie S Price - connie.price@dhha.org; Steve J Morgan - steven.morgan@dhha.org

* Corresponding author

Published: 3 February 2009

Journal of Medical Case Reports 2009, 3:45 doi:10.1186/1752-1947-3-45

This article is available from: http://www.jmedicalcasereports.com/content/3/1/45

(c) 2009 Beieler et al; licensee BioMed Central Ltd.

This is an Open Access article distributed under the terms of the Creative Commons Attribution License (http://creativecommons.org/licenses/by/2.0), which permits unrestricted use, distribution, and reproduction in any medium, provided the original work is properly cited.

\begin{abstract}
Introduction: Multidrug-resistant Acinetobacter baumannii has become a significant cause of healthcareassociated infections, but few reports have addressed Acinetobacter baumannii infections associated with orthopedic devices. The current recommended treatment for complicated infections due to orthopedic devices, including resistant gram-negative rods, consists of antimicrobial therapy with debridement and removal of implants.
\end{abstract}

Case presentation: The patient, a 47-year-old woman, had previously had a prior total hip arthroplasty at 16 years of age for a complex femoral neck fracture, and multiple subsequent revisions. This time, she underwent a fifth revision secondary to pain. Surgery was complicated by hypotension resulting in transfer to the intensive care unit and prolonged respiratory failure. She received peri-operative cefazolin but postoperatively developed surgical wound drainage requiring debridement of a hematoma. Cultures of this grew ampicillin-sensitive Enterococcus and Acinetobacter baumannii (sensitive only to amikacin and imipenem). The patient was started on imipenem. Removal of the total hip arthroplasty was not recommended because of the recent surgical complications, and the patient was eventually discharged home. She was seen weekly for laboratory tests and examinations and, after 4 months of therapy, the imipenem was discontinued. She did well clinically for 7 months before recurrent pain led to removal of the total hip arthroplasty. Intra-operative cultures grew ampicillinsensitive Enterococcus and coagulase-negative Staphylococcus but no multidrug-resistant Acinetobacter baumannii. The patient received ampicillin for 8 weeks and had not had recurrent infection at the time of writing, 37 months after discontinuing imipenem.

Conclusion: We describe the successful treatment of an acute infection from multidrug-resistant Acinetobacter baumannii with debridement and retention of the total hip arthroplasty, using monotherapy with imipenem. This case challenges the general assumption that all orthopedic-device infections due to multidrug-resistant gramnegative organisms will require hardware removal. Further studies are needed to determine if organisms such as multidrug-resistant Acinetobacter baumannii are amenable to treatment with hardware retention. 


\section{Introduction}

Acinetobacter baumannii has become an important cause of healthcare-associated infections worldwide [1]. The organism has developed substantial antimicrobial resistance, making treatment of infections attributed to A. baumannii increasingly more difficult to manage. Multidrugresistant (MDR) A. baumannii is capable of causing a variety of infections, including urinary tract infections, pneumonia, bacteremia, as well as skin and soft tissue infections [2]. It is found primarily in patients with prolonged stays in intensive care units and less commonly in outpatients [3].

A. baumannii has been reported with increasing frequency as a cause of device-associated infections, related to catheters and mechanical ventilation [1,2]. Most recently, MDR A. baumannii has been described as a cause of osteomyelitis in soldiers of the Iraq war [4] and in trauma patients [5].

Despite its increasing frequency, there is little data in the literature on the treatment of orthopedic-device infections due to MDR A. baumannii, specifically on total joint arthroplasties. The recently recommended approach for treating infected arthroplasties is a two-step hardware exchange with use of an antibiotic cement spacer (PROSTALAC) [6]. Here, however, we describe the eradication of MDR A. baumannii total joint arthroplasty infection in a patient using a debridement and retention approach with imipenem-cilastatin (imipenem) monotherapy.

\section{Case presentation}

A 47-year-old woman was involved in a motor vehicle accident at the age of 16 and suffered a right femoral neck fracture. Open reduction internal fixation of the fracture was not possible, and the primary therapy for the injury was a total hip arthroplasty (THA). The patient required four revisions of her primary total hip. She presented to our center for consideration for a fifth revision surgery secondary to intractable pain. She was taken to the operating room (OR) and received peri-operative cefazolin as per protocol. Complications arose during the revision procedure. A femoral shaft fracture occurred, and a deep pelvic arterial injury resulted in intra-operative hemodynamic instability, requiring suspension of the procedure and arterial embolization. The patient was subsequently transferred to the intensive care unit and, on day 2 in hospital, returned to the OR for completion of the original procedure which was uneventful. Her postoperative course, however, was complicated by prolonged respiratory failure necessitating a tracheostomy.

On day 12 in hospital, the patient developed increased pain, redness and new drainage from her surgical wound. She underwent surgical exploration, was found to have a hematoma with evidence of defects in the deep fascia, which required debridement down to the hip prosthesis. The hematoma was evacuated, no antibiotic beads were placed, and she was started on vancomycin. Quantitative cultures were not performed; however, tissue cultures grew ampicillin-sensitive Enterococcus species and A. baumannii (sensitive only to amikacin and imipenem). The patient was switched to imipenem $500 \mathrm{mg}$ IV every 6 hours on day 15 in hospital. She was not considered to be a candidate for removal of her prosthesis because of the recent prior surgical complications.

On day 33 in hospital, the patient returned to the OR for debridement because of continued fevers despite imipenem; cultures once again grew MDR A. baumannii, Enterococcus species and coagulase-negative Staphylococci (CNS). The patient improved and was discharged home on day 53 to complete her imipenem therapy at home.

The patient was seen weekly in our Orthopedic Infectious Diseases Clinic for blood tests and clinical examination. Due to persistently elevated inflammatory markers, erythrocyte sedimentation rate (ESR) and C-reactive protein (CRP), her antibiotic course was extended. Her ESR and CRP improved but never normalized. The antibiotics were stopped after completing a 4-month course of imipenem. The patient was not placed on oral suppressive therapy since no options existed for the MDR A. baumannii.

The patient remained symptom-free for 7 months before noticing pain with walking and swelling at the right hip. She denied having had any new injury or trauma. On exam, she had redness, warmth, swelling and new drainage at the incision site on the right hip, presumed to represent recurrence of her MDR A. baumannii infection. At this time, she underwent surgical debridement and removal of the THA. Intra-operative cultures were obtained but the prosthesis was not sonicated. The operative cultures grew Enterococcus species (sensitive to ampicillin and penicillin), and two types of CNS (resistant to penicillin), which were not thought to be pathogenic. There was no MDR A. baumannii isolated. The patient responded to an 8-week course of therapy directed only toward the Enterococcus species with ampicillin. Twentynine months after stopping antibiotics, she remains free of infection at the time of writing.

\section{Discussion}

Acinetobacter is an aerobic gram-negative coccobacilli that is catalase positive, oxidase negative, non-fermenting and nonmotile [2]. Although it is usually a low-grade pathogen, it has several properties that can increase its virulence. These include a capsule made of polysaccharide (making the organism more hydrophilic), the capability of adhesion to epithelial cells in the company of capsular 
polysaccharide, enzyme production which can injure lipid components of tissue, and possibly a toxic-type function found in the cell wall lipopolysaccharide [2]. Acinetobacter is typically found in environmental areas such as soil and aquatic areas [7]; thus, it can often be a contaminant associated with the environment. However, A. baumannii has recently been linked to hospital infections, including surgical wound infections and device-related infections [1,2].

In a healthcare setting, patients become colonized with the organism on their skin, usually in moist places, the toe webs, axillae and groin [2]. Acinetobacter can live in the hospital setting for several days; it has been demonstrated to survive on filter paper for up to 6 days, on Formica for 13 days and on cotton for 25 days [8]. Risk factors for acquisition of MDR A. baumannii during outbreaks have been described and include the use of broad-spectrum antibiotics, longer hospitalization, advanced age, mechanical ventilation, trauma intensive care unit and the use of pulsatile lavage wound irrigation $[2,9]$.

Before the year 1970, most Acinetobacter species were sensitive to carbenicillin, ampicillin, gentamicin, or minocycline [2]. Around 1975, Acinetobacter was documented as developing resistance to many antibiotics including tetracyclines, cephalosporins, cephamycins, aminopenicillins and aminoglycosides [2]. MDR A. baumannii can be defined as an organism resistant to over three classes of antibiotics [4]. Some A. baumannii are sensitive to newer cephalosporins (ceftazidime), quinolones, amikacin, tobramycin and imipenem, but often with higher minimum inhibitory concentrations than before [2].

Recently, increases in imipenem-resistant MDR A. baumannii infections have lead to the use of colistin or polymyxin B for salvage therapy in severe cases [10]. Colistin and polymyxin $B$ have proven to be successful treatments of MDR A. baumannii bacteremia, pneumonia, meningitis and orthopedic-device infections in documented case reports [10].

Recent cases of MDR A. baumannii infections in military personnel have occurred in the form of bacteremia, extremity war wounds and osteomyelitis [4]. In these cases, osteomyelitis was documented via bone cultures taken intra-operatively during open debridements or internal/external fixation procedures [4]. Other patients with symptoms of fever, increased white blood cell count, open fractures with necrotic tissue and gross pus with deep tissue cultures positive for MDR A. baumannii were also classified as osteomyelitis [4]. In the military patients treated for osteomyelitis, ampicillin/sulbactam, ceftazidime and amikacin in combination with imipenem were used successfully [4]. The duration of therapy in this small study of 18 patients varied between 4 to 8 weeks of IV treatment [4]. In a mean follow-up time of 9 months, there were no recurrences of MDR A. baumannii infection [4].

Previously published guidelines related to orthopedic device infections suggest that prerequisites for debridement and retention include short duration of symptoms, satisfactory condition of soft tissue and the absence of difficult-to-treat resistant microorganisms [6], particularly gram-negative organisms [11]. These guidelines, as they relate to gram-negative pathogens such as A. baumannii as well as MDR phenotypes, are based on little evidence and mostly on speculation. This is due, in part, to the inability to confirm microbiologic eradication of the offending pathogen. In our patient's case, microbiologic eradication was documented in a subsequent debridement several months after antibiotics had been discontinued.

New information suggests that $A$. baumannii is capable of forming biofilms on different surfaces, which may explain in part why these infections are difficult to treat. A. baumannii biofilms that adhere to various glass (hydrophilic) and plastic (hydrophobic) surfaces were observed in recent studies [12]. After allowing the organism to set for approximately 10 to 15 minutes, the biofilm made by the A. baumannii 19606 strain was shown to remain on both Teflon strips and Petri dishes after washing with tap water [12]. The A. baumannii biofilm demonstrated growth in both static and dynamic environments and at varying temperatures, verifying that an A. baumannii organism can persevere and multiply in harsh conditions [12]. This information suggests that device-related infections due to A. baumannii require definitive antibiotic therapy and, if treatment fails to be effective, possible device removal for cure.

\section{Conclusion}

A. baumannii continues to emerge as an important cause of trauma-associated infections and orthopedic deviceassociated infections. Consequently, more evidence is necessary to understand the importance of gram-negative infections and MDR phenotypes, especially MDR A. baumannii, and factors associated with failure when salvage is attempted. It is interesting that the more sensitive grampositive organism (ampicillin-sensitive Enterococcus) persisted in our patient despite active therapy against this organism, again calling into question the assumption that gram-negative organisms are inherently more difficult to eradicate.

We described a case report of successful treatment of a MDR A. baumannii infected THA with debridement and retention, and 4 months of monotherapy using imipenem, with microbiologic eradication of MDR A. bau- 
mannii, and no signs of recurrence with this organism at 37 months. Assumptions that orthopedic device infections due to gram-negative organisms and/or those with multidrug-resistant phenotypes are not amenable to salvage should be re-examined.

\section{Abbreviations}

MDR: Multidrug-resistant; THA: total hip arthroplasty; OR: operating room; CNS: coagulase-negative Staphylococcus; ESR: erythrocyte sedimentation rate; CRP: C-reactive protein.

\section{Consent}

The patient gave verbal consent to have her case written up for a published medical report. Due to mobility issues the patient preferred to give verbal consent for this case presentation. Documentation of this consent is available for review by the Editor-in-Chief of this journal.

\section{Competing interests}

The authors declare that they have no competing interests.

\section{Authors' contributions}

$\mathrm{AMB}$ was the provider who assisted in caring for the patient while on antibiotic therapy, obtained consent, obtained chart records, prepared the manuscript, and submitted the manuscript for publication. RWB was the Infectious Disease specialist who assisted in caring for the patient, and contributed to manuscript editing for medical accuracy and content. MRD was the Orthopedic surgeon caring for the patient, and assisted with manuscript editing for medical accuracy. CSP was the Infectious Disease specialist who assisted in caring for the patient and contributed to manuscript editing for medical accuracy and content. SJM was the Orthopedic surgeon caring for the patient, and assisted with collection of chart records, and manuscript editing for medical accuracy.

\section{References}

I. Corbella X, Montero A, Pujol M, Dominguez MA, Ayats J, Argerich MJ, Garrigosa F, Ariza J, Gudiol F: Emergence and rapid spread of carbapenem resistance during a large and sustained hospital outbreak of multiresistant Acinetobacter baumannii. J Clin Microbiol 2000, 38(I I):4086-4095.

2. Bergogne-Berezin E, Towner KJ: Acinetobacter spp. as nosocomial pathogens: microbiological, clinical, and epidemiological features. Clin Microbiol Rev 1996, 9(2): |48-165.

3. Fridkin SK, Steward CD, Edwards JR, Pryor ER, McGowan JE Jr, Archibald LK, Gaynes RP, Tenover FC: Surveillance of antimicrobial use and antimicrobial resistance in United States hospitals: Project ICARE Phase 2. Clin Infect Dis 1999, 29(2):245-252.

4. Davis KA, Moran KA, McAllister CK, Gray PJ: Multidrug-resistant Acinetobacter extremity infections in soldiers. Emerg Infect Dis 2005, I I (8): I 2 | 8- I 224.

5. El Shafie SS, Alishaq M, Leni Garcia M: Investigation of an outbreak of multidrug-resistant Acinetobacter baumannii in trauma intensive care unit. J Hosp Infect 2004, 56(2): I0I-105.

6. Zimmerli W, Trampuz A, Ochsner PE: Prosthetic-joint infections. N Engl J Med 2004, 35 I( I 6): I 645- I 654.

7. Baumann P: Isolation of Acinetobacter from soil and water. J Bacteriol 1968, 96(I):39-42.
8. Wendt C, Dietze B, Dietz E, Ruden H: Survival of Acinetobacter baumannii on dry surfaces. J Clin Microbiol I 997, 35(6): I 394- I 397.

9. Maragakis LL, Cosgrove SE, Song X, Kim D, Rosenbaum P, Ciesla N, Srinivasan A, Ross T, Carroll K, Perl TM: An outbreak of multidrug-resistant Acinetobacter baumannii associated with pulsatile lavage wound treatment. JAMA 2004, 292(24):3006-30I I.

10. Li J, Nation RL, Turnidge JD, Milne RW, Coulthard K, Rayner CR, Paterson DL: Colistin: the re-emerging antibiotic for multidrug-resistant gram-negative bacterial infections. Lancet Infect Dis 2006, 6(9):589-60I.

II. Tsukayama DT, Estrada R, Gustilo RB: Infection after total hip arthroplasty. A study of the treatment of one hundred and six infections. J Bone Joint Surg Am 1996, 78(4):5 I 2-523.

12. Tomaras AP, Dorsey CW, Edelmann RE, Actis LA: Attachment to and biofilm formation on abiotic surfaces by Acinetobacter baumannii: involvement of a novel chaperone-usher pili assembly system. Microbiol 2003, I 49( I 2):3473-3484.
Publish with Bio Med Central and every scientist can read your work free of charge

"BioMed Central will be the most significant development for disseminating the results of biomedical research in our lifetime. "

Sir Paul Nurse, Cancer Research UK

Your research papers will be:

- available free of charge to the entire biomedical community

- peer reviewed and published immediately upon acceptance

- cited in PubMed and archived on PubMed Central

- yours - you keep the copyright

Submit your manuscript here:

http://www.biomedcentral.com/info/publishing_adv.asp
BioMedcentral 